\title{
Una nueva especie de spheniscidae del Mioceno Tardío de la Formación Pisco, Perú
}

Une nouvelle espèce de spheniscidae du miocène supérieur de la Formation pisco, Pérou

A new species of spheniscidae from the Late Miocene of the Pisco Formation, Peru

\section{Marcelo Stucchi, Mario Urbina y Alfredo Giraldo}

\section{OpenEdition}

\section{Journals}

Edición electrónica

URL: http://journals.openedition.org/bifea/6541

DOI: 10.4000/bifea.6541

ISSN: 2076-5827

\section{Editor}

Institut Français d'Études Andines

\section{Edición impresa}

Fecha de publicación: 1 septiembre 2003

Paginación: 361-375

ISSN: 0303-7495

\section{Referencia electrónica}

Marcelo Stucchi, Mario Urbina y Alfredo Giraldo, « Una nueva especie de spheniscidae del Mioceno Tardío de la Formación Pisco, Perú », Bulletin de l'Institut français d'études andines [En línea], 32 (2) 2003, Publicado el 08 agosto 2003, consultado el 01 diciembre 2020. URL : http:// journals.openedition.org/bifea/6541; DOI : https://doi.org/10.4000/bifea.6541

\section{(c) $(1) \odot$}

Les contenus du Bulletin de l'Institut français d'études andines sont mis à disposition selon les termes de la licence Creative Commons Attribution - Pas d'Utilisation Commerciale - Pas de Modification 4.0 International. 


\title{
UNA NUEVA ESPECIE DE SPHENISCIDAE DEL MIOCENO TARDÍO DE LA FORMACIÓN PISCO, PERÚ
}

\author{
Marcelo STUCCHI*, Mario URBINA*, Alfredo GIRALDO***
}

\section{Resumen}

Se describen elementos craneales atribuibles a una nueva especie del género Spheniscus, procedentes del Mioceno tardío de la Formación Pisco, en la costa centro-sur del Perú. Esta nueva especie se caracteriza por tener un rostrum, en relación con el cráneo, proporcionalmente mayor que el observado en las demás especies del género. Finalmente, se discute brevemente sobre el valor adaptativo de los caracteres observados.

Palabras claves: Spheniscidae, Sphenisciformes, Spheniscus, Formación Pisco, Perú, Mioceno Tardío.

\section{UNE NOUVELLE ESPÈCE DE SPHENISCIDAE DU MIOCÈNE SUPÉRIEUR DE LA FORMATION PISCO, PÉROU}

\section{Résumé}

Nous décrivons des éléments crâniens attribués à une nouvelle espèce du genre Spheniscus, provenant du Miocène supérieur de la Formation Pisco, sur la côte centre-sud du Pérou. Cette nouvelle espèce se caractérise par la présence d'un rostrum proportionnellement plus grand que celui observé chez les autres espèces du genre. Finalement, l'on discute brièvement sur la valeur adaptative des caractères observés.

Mots clés : Spheniscidae, Sphenisciformes, Spheniscus, Formation Pisco, Pérou, Miocène supérieur.

\section{A NEW SPECIES OF SPHENISCIDAE FROM THE LATE MIOCENE OF THE PISCO FORMATION, PERU}

\section{Abstract}

A new species of genus Spheniscus is described based on cranial elements from Late Miocene of Pisco Formation on the central-southern coast of Peru. The skull to rostrum

* Departamento de Paleontología de Vertebrados. Museo de Historia Natural - Universidad Nacional Mayor de San Marcos, Lima (Perú). E-mail: marstuc@yahoo.com

** Universidad Nacional Agraria La Molina, Lima (Perú). 
proportion of this new species is bigger than any other species of the genus. Finally, the adaptative value of the observed characteristics is briefly discussed.

Key words: Spheniscidae, Sphenisciformes, Spheniscus, Pisco Formation, Peru, Late Miocene.

\section{INTRODUCCIÓN}

Hasta el momento no se había realizado un estudio detallado del material de Spheniscidae procedente de la Formación Pisco (Mioceno tardío-Plioceno temprano) en la costa centro-sur del Perú (Fig. 1). El material descrito previamente está clasificado de forma preliminar en varias categorías distintas; dentro del género Spheniscus, como Spheniscidae indeterminado (pero diferente a Spheniscus) (Marocco \& Muizon, 1988) y como próximo a los géneros Eudyptes y Spheniscus (Noriega \& Tambussi, 1989).

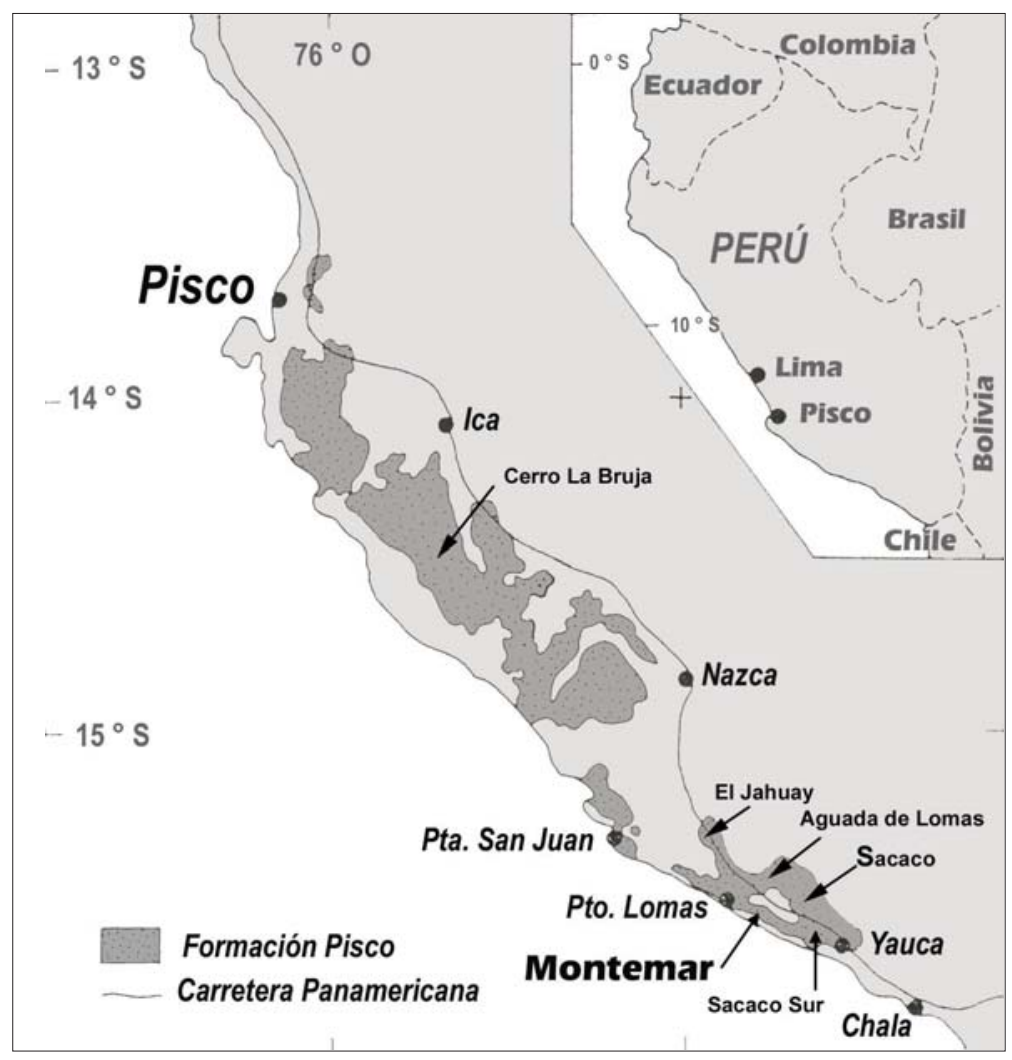

Fig. 1 - Mapa de la Formación Pisco. Tomado de Muizon \& DeVries (1985). 
El registro fósil de los Sphenisciformes está compuesto por al menos 11 géneros paleontológicos y 3 actuales. Este se remonta al Paleoceno tardío (Thaetiano, 55 - 56 Ma) de la Antártida, con restos que pertenecieron a una forma de grandes dimensiones (Tambussi et al., 2002). Para el Eoceno tardío de Nueva Zelanda y de la Isla Vicecomodoro Marambio (Seymour) en la Antártida (45-35 Ma) se incluyen a las especies de mayor tamaño conocidas hasta el momento y otras de tamaño medio (géneros Anthropornis, Palaeeudyptes, Archaeospheniscus, Delphinornis, Mesetaornis y Marambiornis) (Myrcha et al., 2002).

El lapso comprendido entre el Oligoceno tardío-Mioceno temprano en la Patagonia (Argentina) da cuenta de otro importante evento, encontrándose allí los restos de 7 especies pertenecientes a los géneros Microdytes, Chubutodyptes, Paraptenodytes y Palaeospheniscus (Tonni \& Tambussi, 1986). Estos dos últimos se mantienen al menos hasta el Mioceno tardío-Plioceno temprano de Chile (Acosta et al., 2002). Entre estos géneros, es Palaeospheniscus el que morfológicamente se acerca más a las especies actuales. Sin embargo, marcadas diferencias en el tarsometatarso se usaron para proponer que el género podría pertenecer a un linaje paralelo (Simpson, 1946: 70). También del Plioceno proviene Marplesornis novaezealandie de Nueva Zelanda, asignado inicialmente al género Palaeospheniscus (Marples, 1960).

Los registros atribuibles a géneros actuales son mucho más recientes y se reconocen en el Mioceno tardío de la Fm. Pisco de Perú (Marocco \& Muizon, 1988) y de la Fm. Bahía Inglesa de Chile (Walsh \& Hume, 2001), referidos al género Spheniscus. Del Plioceno temprano-Pleistoceno de la Provincia de El Cabo (Sudáfrica) se han reportado restos considerados por Olson (1983: 399) como posibles formas primitivas de Spheniscus; una de ellas nominada por Simpson(1971) como Spheniscus predemersus. Del Plioceno tardío de Nueva Zelanda están citadas especies de los géneros Aptenodytes y Pygoscelis (Simpson, 1976: 67), este último género también conocido en el Mioceno tardío-Plioceno temprano de Chile (Acosta et al., 2002).

Recientes hallazgos realizados en los depósitos marinos de la Fm. Pisco, permiten reconocer una nueva especie del género Spheniscus, sobre la base de un cráneo completo, dos parciales y dos mandíbulas.

\section{SISTEMÁTICA PALEONTOLÓGICA}

Orden Sphenisciformes Sharpe, 1891

Familia Spheniscidae Bonaparte, 1831

Género Spheniscus Moehring, 1758

\section{1. Spheniscus megaramphus sp. nov.}

(Figs. 2 - 8)

\section{1. 1. Etimología}

Megas (griego) grande y Ramphos (griego) pico; en referencia al tamaño del pico proporcionalmente mayor que las demás especies del género. 


\section{1. 2. Holotipo}

MUSM 175: Neurocráneo separado del rostrum. Sin los lacrimales, la región de los procesos nasales del premaxilar, los yugales, los pterigoides, los cuadrados, los palatinos, parte del pleuroesfenoides, el plato basitemporal y rostrum paraesfenoidal. Mandíbula fragmentada y dentarios parciales (a nivel del proceso coronoides).

\section{1. 3. Paratipos}

MUSM362: Neurocráneo incompleto y porción articular derecha de la mandíbula.

MUSM 363: Neurocráneo incompleto y rostrum sin porción anterior.

MUSM 364: Rama mandibular derecha y fragmento de rama izquierda.

MUSM 365: Rama mandibular izquierda y fragmento de rama derecha. Parte anterior del rostrum y fragmento de techo craneano.

\section{1. 4. Procedencia geográfica y estratigráfica}

Los especímenes proceden del nivel bioestratigráfico de Montemar, el cual de acuerdo a Marocco \& Muizon (1988) tiene una antigüedad de 6 Ma (Mioceno tardío). Devries (comp. pers., 2002) sin embargo, sugiere que es posible que este nivel sea al menos 1,5-1,6 Ma menor.

Todos han sido colectados por Mario Urbina en 2000. El material pertenece a la Colección del Departamento de Paleontología de Vertebrados del Museo de Historia Natural, Universidad de San Marcos (Lima, Perú).

\section{1. 5. Material de comparación}

El material de comparación consiste en 50 cráneos de la especie actual Spheniscus humboldti, 2 de $S$. magellanicus, 2 de $S$. mendiculus y 1 de $S$. demersus.

El material de las especies $S$. humboldti y $S$. magellanicus revisado pertenece a la Colección de Referencia del Departamento de Paleontología de Vertebrados del Museo de Historia Natural, Universidad de San Marcos (Lima, Perú).

El material de S. mendiculus pertenece al Museo de Zoología de la Pontificia Universidad Católica del Ecuador (QCAZ 1218, QCAZ 1223) y el de S. demersus al South African Museum de Ciudad El Cabo (Zo 58106).

\section{1. 6. Diagnosis}

Representante del género Sphenfiscus hasta $30 \%$ más grande que las especies actuales. Se diferencia de estas por poseer el rostrum $60 \%$ más largo en relación al neurocráneo y proporcionalmente más angosto a nivel de la sutura fronto-nasal. Techo craneano reducido. Mandíbula recta con articular más corto y robusto que en las demás especies del género. 


\section{DESCRIPCIÓN}

\section{1. El rostrum}

El rostrum presenta una superficie rugosa formada por diminutos orificios, en las caras lateral y ventral de su extremo anterior. Este es bastante más largo que la caja craneana. El índice 1 (Cuadro 1) muestra la relación de tamaño entre el rostrum y el cráneo de $S$. megaramphus y los miembros actuales del género, quienes mantienen una proporción casi igual entre ambos elementos.

En vista lateral (Fig. 2) el rostrum es recto dorsal y ventralmente curvándose sólo en el extremo anterior para formar la punta. Ventralmente, esta curvatura representa sólo una octava parte de su longitud, a diferencia de los actuales donde representa aproximadamente una sexta parte. La punta, en forma de gancho, es proporcionalmente más grande que en las formas actuales. Las aberturas nasales son bastante amplias y de forma cónica, notoriamente comprimidas en el sentido dorsoventral, lo que caracteriza al género. Su límite posterior se encuentra ligeramente por delante que el límite anterior de la fosa preorbitaria, como en las especies actuales.

En vista dorsal (Fig. 3) al igual que en los actuales, se aprecia un estrechamiento desde la base del rostrum (sutura fronto-nasal) en sentido anterior o craneal, siendo el ancho al nivel del límite posterior de la fosa nasal cercano al doble de aquél observado en su límite anterior.

En vista ventral, la fusión de los maxilares en el extremo anterior representa sólo la séptima parte de la longitud del rostrum, en cambio en los actuales ocupa casi la tercera parte de dicha longitud. Los maxilares y palatinos están longitudinalmente separados (paladar esquizognato). En MUSM 362 se aprecia el palatino derecho, de igual constitución que en las especies actuales del género (Fig. 4). Se pueden apreciar además dos grandes y curvos procesos maxilopalatinos que se prolongan hasta la sutura frontonasal (Fig. 5).

\section{2. El neurocráneo}

El neurocráneo mantiene las mismas proporciones que los actuales (ver Índice 2, Cuadro 1). Los frontales (Figs. 3 y 4) son muy reducidos en comparación con los de las especies actuales. Forman un techo craneano de superficie lisa y comprimido en el sentido dorsoventral, distinto del techo abovedado observado en la mayoría de los ejemplares de las especies actuales.

Dorsalmente, se aprecian las depresiones supraorbitales, de superficie rugosa y prolongadas posteriormente hasta el nivel de los procesos postorbitales. A este nivel se hacen más profundas, como en algunos ejemplares de S. magellanicus y de S. humboldti. En S. megaramphus, dichas depresiones son mucho mayores que en las especies actuales, a tal punto que sus límites posteriores están separados de las fosas temporales solamente por una cresta muy angosta del frontal. Este último carácter puede apreciarse en algunos especímenes de $S$. magellanicus y $S$. humboldti, pero por lo general dicha región es lisa y redondeada. La separación de las depresiones supraorbitales y de las fosas temporales es más ancha antero - posteriormente. 
Cuadro 1- Comparación entre las medidas e índices de las especies del genero Spheniscus.

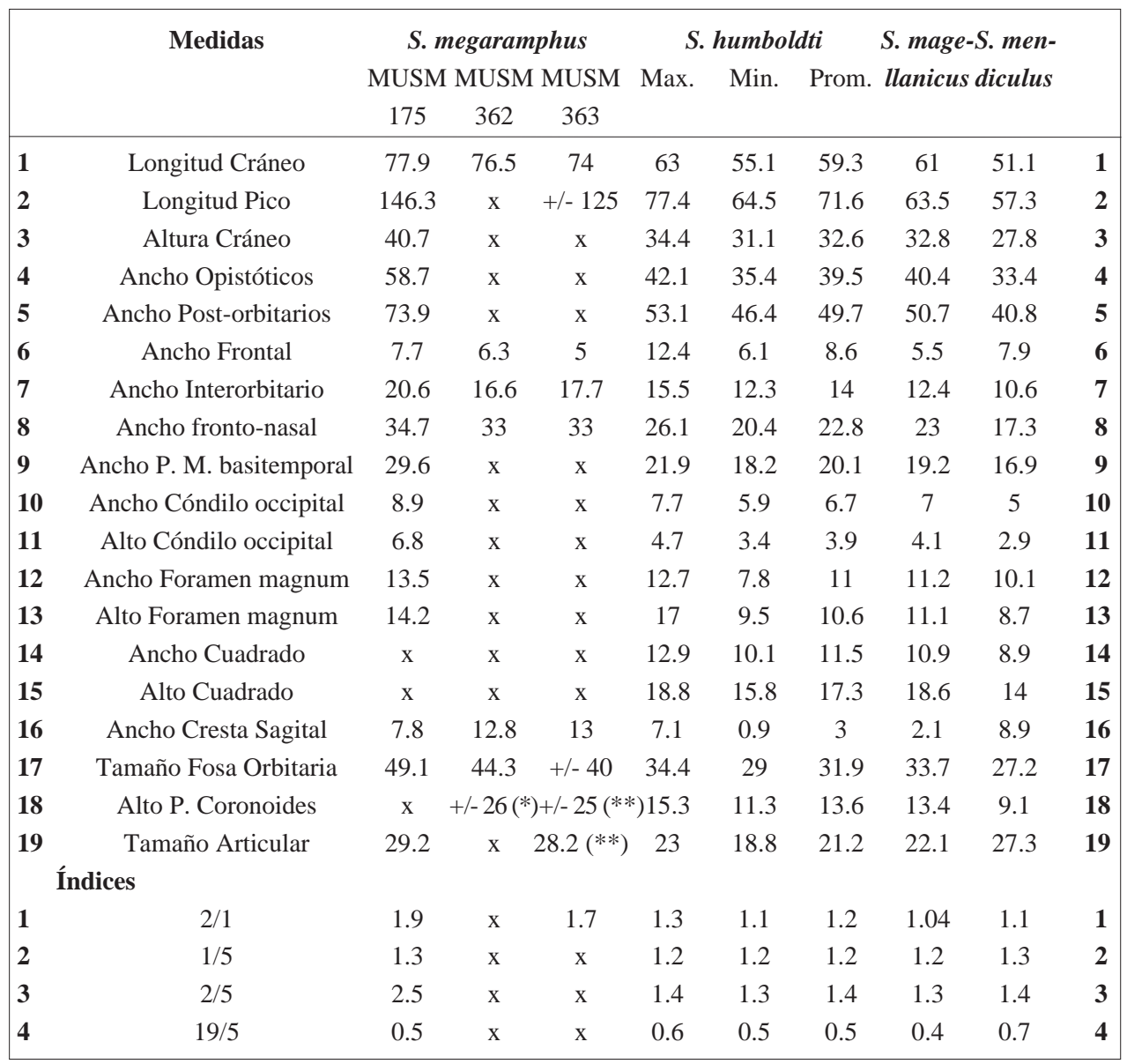

S. humboldti, $\mathrm{n}=5$ (『. magellanicus y $S$. mendiculus, $\mathrm{n}=2$.

Sólo para S. humboldti: la medida $\mathrm{N}^{\mathrm{o}} 2$ correspondiente a la longitud del pico, fue hecha con la presencia de la ranfoteca siguiendo el criterio utilizado para medir pingüinos vivos (Zavalaga \& Paredes 1997: 104-105). La medida en los fósiles y las otras especies actuales se hace desde la sutura fronto-nasal. Bajo este mismo principio, la longitud del pico es $3 \mathrm{~mm}$ menor que el promedio indicado. Este valor fue obtenido con $\mathrm{n}=36$.

(*) esta medida corresponde a MUSM 364

(**) estas medidas corresponden a MUSM 365

Índice 3: Grado de elongación del pico: Valor para A. patagonica $=2,4$

Índice 4: Movilidad mandibular: Valor para A. patagonica $=0,36$ (Según Zusi 1975: 67)

Explicación de algunas medidas

6. se mide a $15 \mathrm{~mm}$ de la sutura Fronto-nasal.

7. se mide a la misma altura que la medida anterior.

14. en el área que contacta con el articular

17. desde el proceso pre-orbitario hasta el límite anterior del proceso post-orbitario. 


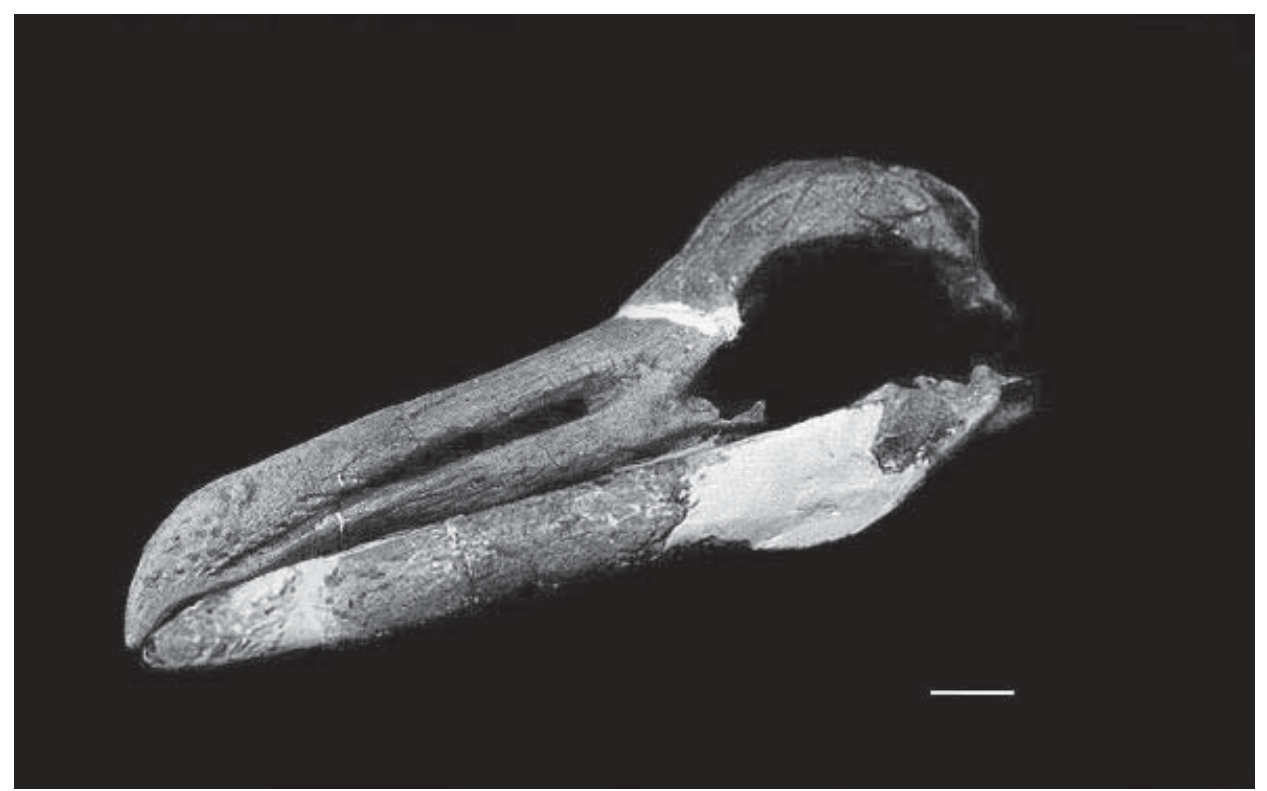

Fig. 2 - S. megaramphus sp. nov. MUSM 175. El neurocráneo y el rostrum han sido unidos en función al plano basal, según se aprecia en MUSM 363. La mandíbula está reconstruida sobre la base de MUSM 364. Las escalas de cada figura indican 20 mm.

Los procesos postorbitales son de tamaño pequeño, en relación con los observados en las formas actuales. Sin embargo, se disponen de modo similar ya que su límite anterior se ubica dorsalmente y el posterior ventralmente, siguiendo la forma de la órbita. Entre ambos procesos postorbitales se encuentra el ancho mayor del cráneo.

La órbita es grande y hasta alrededor de un $30 \%$ mayor que en los actuales, pero manteniendo la misma proporción con respecto al tamaño del neurocráneo. Entre ambas órbitas se forma el septo interorbitario, del cual queda sólo un pequeño fragmento. Anterior a este, se observan los canales óseos nasales de forma tubular.

Los parietales forman lateralmente la fosa temporal donde se aloja el músculo aductor mandibular externo, que se inserta dorsalmente sobre la cresta sagital (Fig. 3 y 4). Dicha cresta comprende el punto de máxima aproximación entre ambas fosas, cruzado por la línea media del cráneo. Las paredes parietales de las fosas son superficies convexas en forma de bóveda, pero menos dilatadas que en los actuales. MUSM 362 y MUSM 363 las presentan más dilatadas que el holotipo, tanto como los actuales. Fosas temporales más profundas y más cortas antero - posteriormente que en los actuales.

Los escamosos se prolongan lateralmente formando una plataforma horizontal al igual que en los actuales y presentan un reducido proceso zigomático.

La región occipital es de forma trapezoidal con los bordes laterales y superiores formados por las crestas post-temporales, las cuales son bastante más amplias que en MUSM 362, MUSM 363 y los actuales. La prominencia cerebelaris es muy poco 


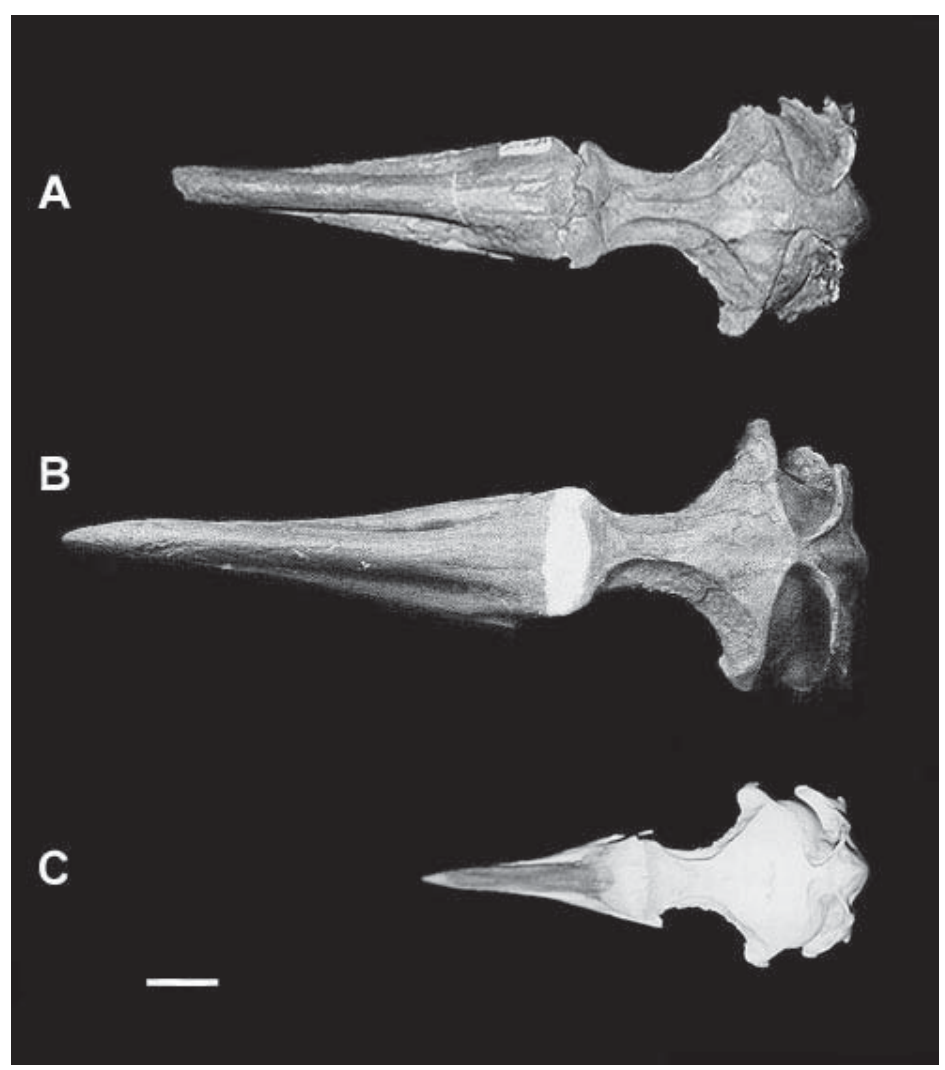

Fig. 3 - A: S. megaramphus sp. nov. MUSM 363; B: S. megaramphus sp. nov. MUSM 175. C: $S$. humboldti. Vista dorsal.

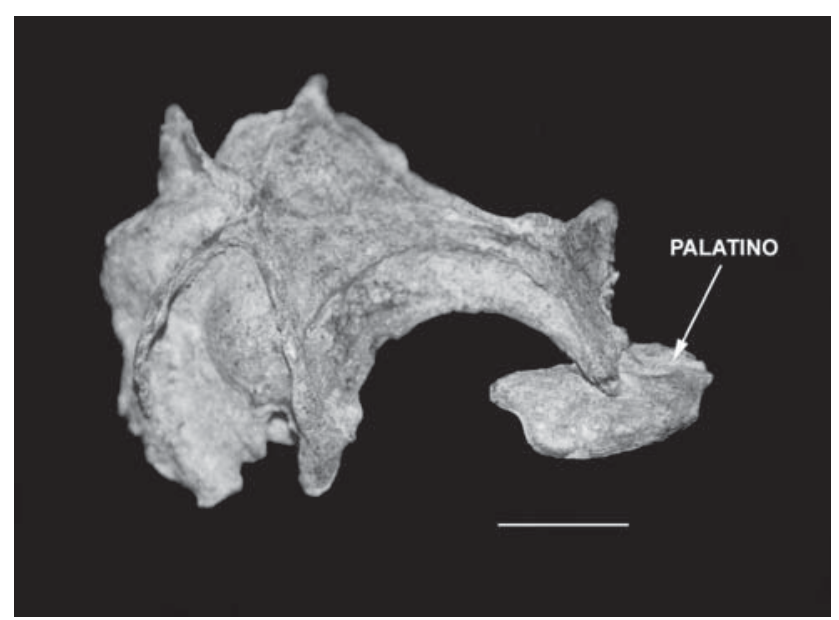

Fig. 4 - A: S. megaramphus sp. nov. MUSM 362. Vista dorsal. 


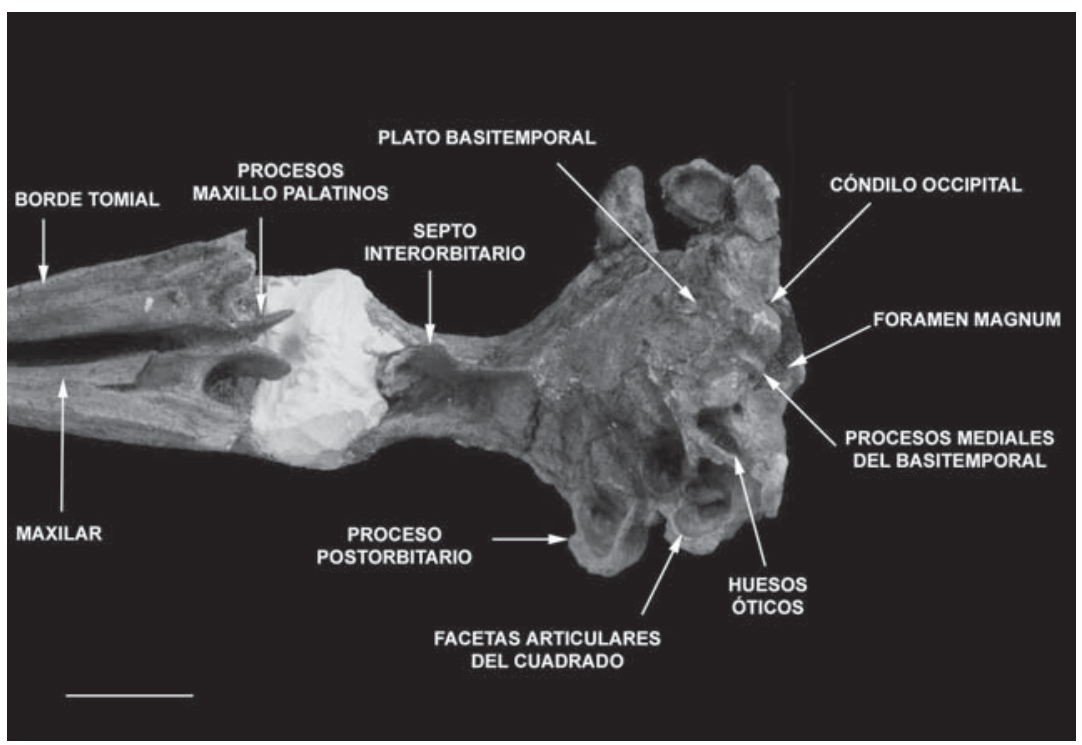

Fig. 5 - S. megaramphus sp. nov. MUSM 175. Vista ventral.

desarrollada en el holotipo y MUSM 363 en comparación con MUSM 362 y las especies actuales. Lateralmente a la prominencia cerebelaris se encuentran los epióticos formando depresiones (occipital fonticulus) a ambos lados de éste. Allí se ubica el foramen de la vena occipital, y se prolonga sobre la superficie del hueso en forma de canal como se aprecia en la mayoría de individuos de $S$. humboldti. El foramen magnum presenta la misma disposición que en las actuales, de forma ovoide con el límite dorsal más angosto y proyectado posteriormente a su límite ventral.

El cóndilo occipital es de forma elíptica comprimido dorsoventralmente, al igual que en los actuales.

Los exoccipitales limitan ventralmente con el plato basitemporal, en cuyos límites se forman en cada lado los procesos mediales del basitemporal y se presentan como dos pequeñas salientes ventrales a los lados del cóndilo. En el occiput del cráneo forman, adyacentes a los opistóticos, los límites laterales inferiores del foramen magnum. Los opistóticos se presentan no bífidos y se orientan ventralmente.

En la porción ventral del neurocráneo (Fig. 5) se aprecia que los huesos óticos están fusionados. El plato basitemporal, observable sólo por la impresión en el sedimento, es plano y tiene forma hexagonal. La fosa pneumática es relativamente grande, muy próxima a las facetas articulares del cuadrado. Dichas facetas están separadas por la abertura timpánica superior, en un grado proporcional al observado en los actuales. La cavidad timpánica es grande con su pared en forma cónica. Se aprecia el proceso metótico, donde se ven los forámenes de nervios IX y X, además los forámenes de los nervios XI, XII, el foramen carotideo, el foramen proóticum y la fenestra ovalis. 


\section{3. La mandíbula}

Como se aprecia en MUSM 364 y MUSM 365, los dentarios son distintos que en las especies actuales (Fig. 6). Se presentan rectos a lo largo de toda la mandíbula, curvándose sólo a la altura donde termina la inserción de la ranfoteca, al igual que los actuales, pero de forma mucho menos pronunciada. En su extremo anterior presenta una superficie lateral rugosa formada por diminutos agujeros, mientras que en su superficie medial se observa a lo largo de la misma, la depresión donde se inserta la ranfoteca. La sínfisis mandibular es pequeña, presentando en su aspecto dorsal una hendidura a manera de estuche donde encaja la punta del rostrum.

Al igual que en las especies actuales, la región articular presenta dos procesos: post-articular e interno. Sin embargo, posee características que lo diferencian de estos:

- En vista dorsal (Fig. 7) se aprecia que el proceso postarticular es más corto y robusto; está separado del proceso interno por una superficie cóncava, aunque no tan profunda como los actuales. Asimismo, se aprecia la superficie articular más angosta antero-posteriormente que en los actuales.

-En vista medial (Fig. 8) se observa que el proceso articular interno es corto y en forma de gancho ascendente, pero más bajo que en los actuales. En esta misma vista, se aprecia que el proceso postarticular es más corto y ancho, presentando una concavidad bien marcada en el extremo opuesto del borde que lo une al proceso interno (Fig. 8a). $\mathrm{Su}$ superficie ventral es recta, siendo muy curva en los actuales. En vista externa (Fig. 6) se aprecia mejor la diferencia entre el proceso postarticular de S. megaramphus y los actuales.

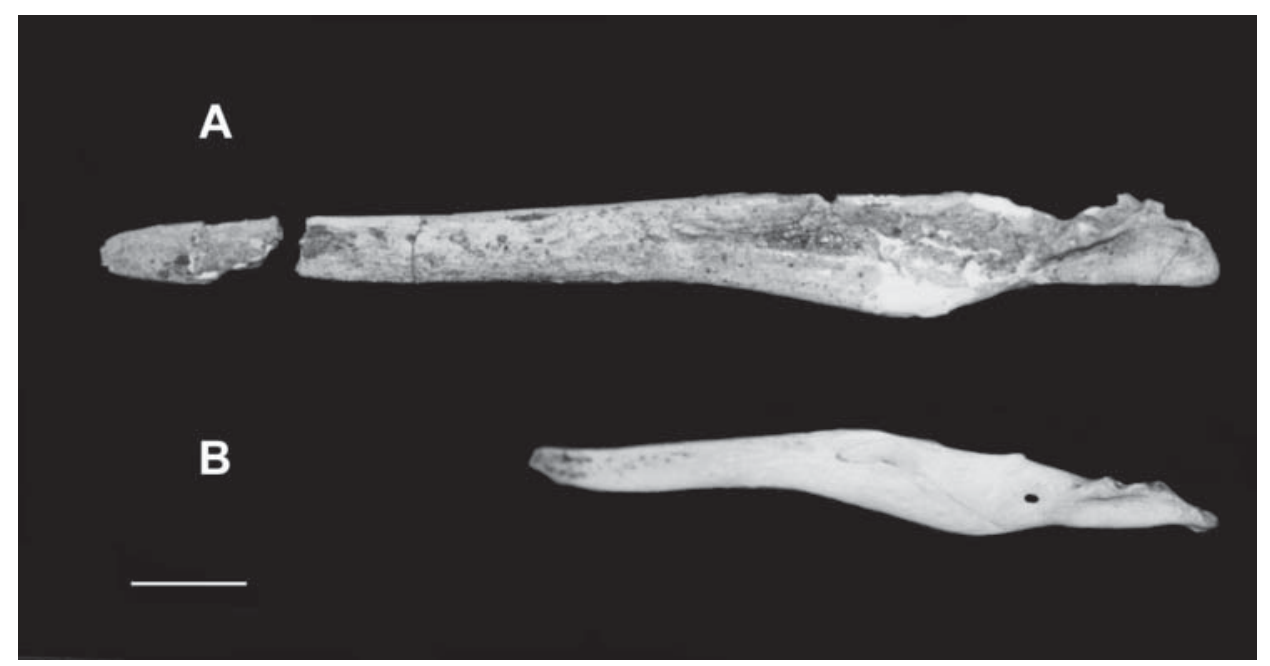

Fig. 6 - A: S. megaramphus sp. nov. MUSM 364; B: S. humboldti. Vista lateral externa del dentario derecho. Nótese el articular. 


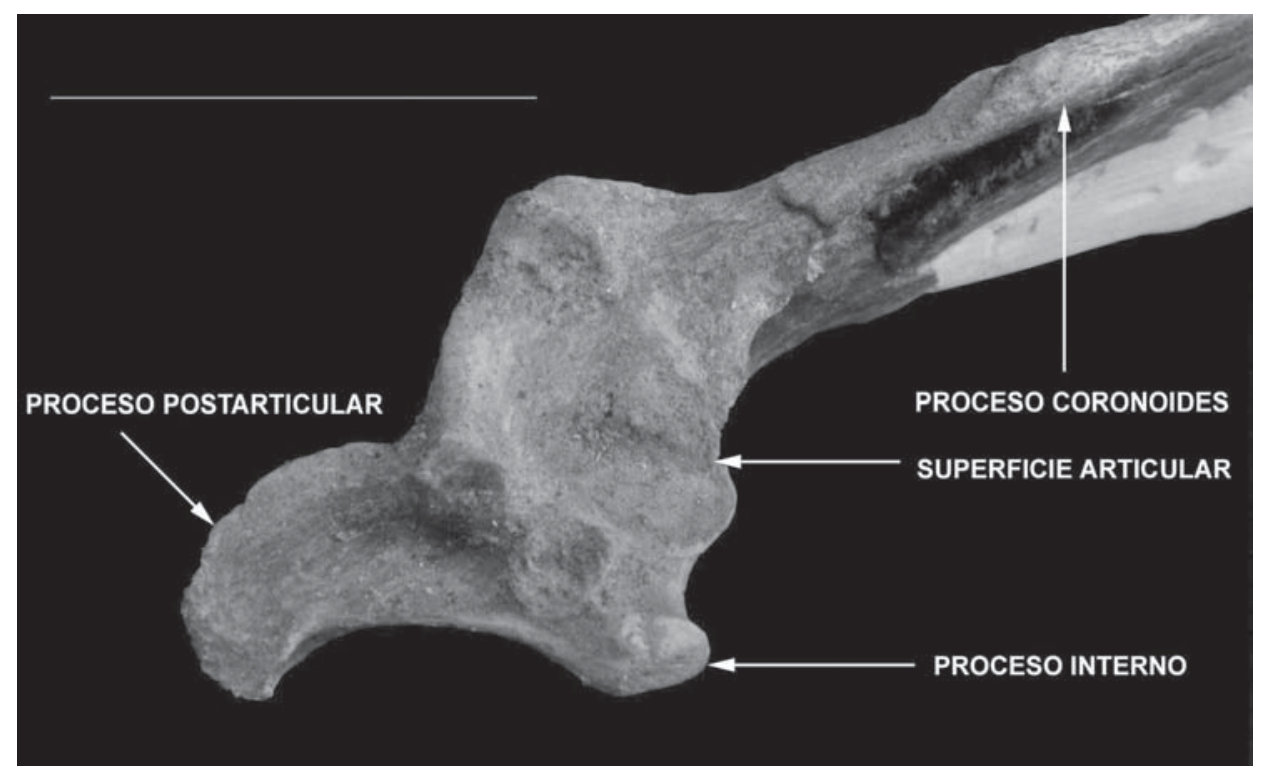

Fig. 7 - S. megaramphus sp. nov. MUSM 175. Vista dorsal del articular.

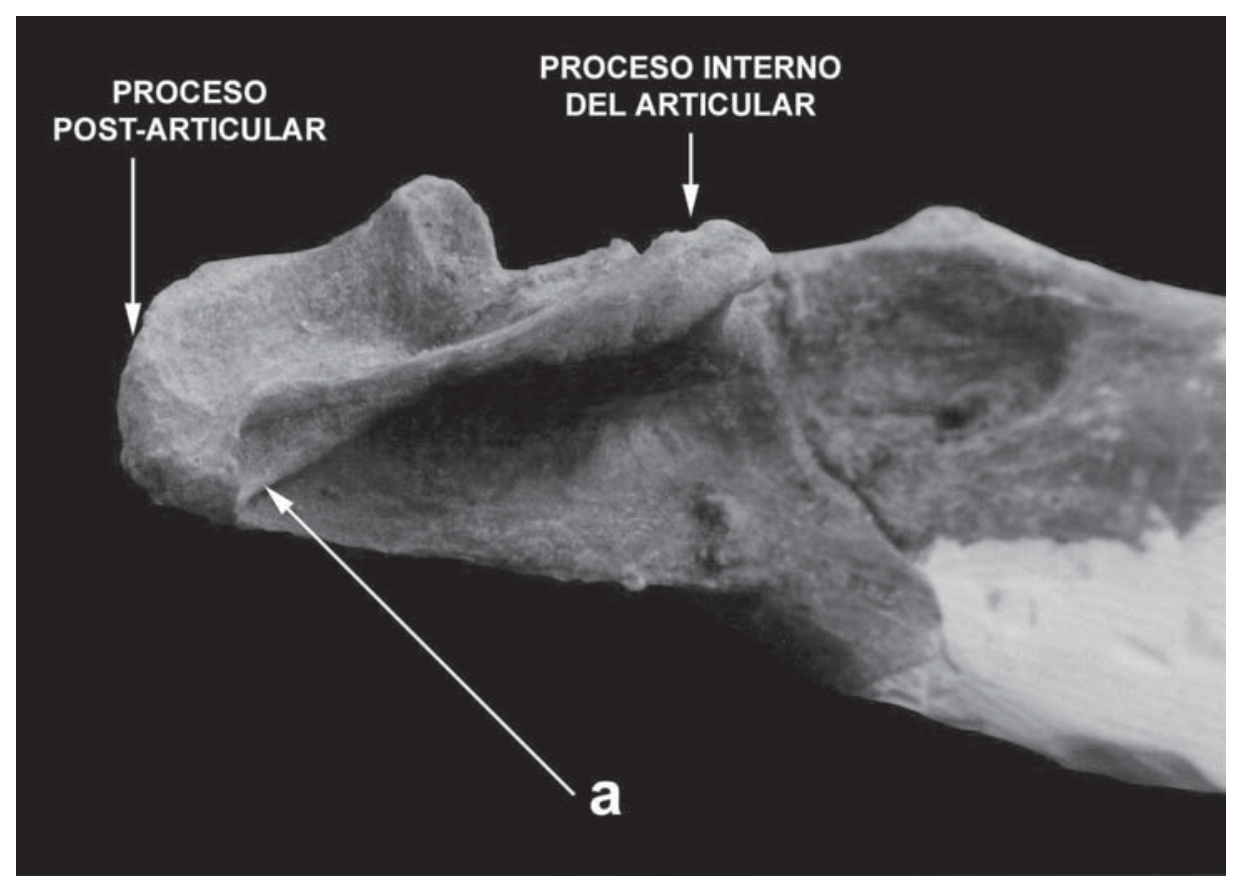

Fig. 8 - S. megaramphus sp. nov. MUSM 175. Vista medial del articular. 


\section{DISCUSIÓN}

\section{1. Determinación del género y la especie}

Según Zusi (1975) el género Spheniscus está definido por los siguientes caracteres craneales (ver Zusi 1975: 61-63, figs. 4.1 y 4.2): exclusivo para Spheniscus: (1) presencia de un proceso postarticular muy grande, con su superficie marcadamente cóncava entre este y el proceso articular interno.

Compartido con los géneros fósiles Paraptenodytes y Marplesornis: (2) presencia de una cresta sagital en la línea media del cráneo, donde se encuentran (o casi se encuentran) las dos fosas temporales.

Compartidos con el género Eudyptula: (3) barra yugal ligeramente curva; (4) maxila con borde tomial vertical.

Compartidos con los géneros Eudyptula, Eudyptes y Megadyptes: (5) proceso postarticular angosto en vista dorsal y más pequeño que la superficie articular; (6) borde tomialde la mandíbula superior aproximadamente al mismo nivel del plato basitemporal; y (7) músculo aductor mandibular externo ocupando la mayor parte de la superficie lateral de la mandíbula.

Una octava característica para el género Spheniscus, que se desprende del estudio de Zusi (1975: 67) consiste en el mayor valor para el índice de movilidad mandibular, donde este género presenta un promedio siempre mayor a 0,45 a diferencia del resto de géneros.

Todos los caracteres citados son verificables en S. megaramphus a excepción del tercero, porque ambas barras yugales están ausentes. El séptimo sólo podría ser deducido estimando el grado de desarrollo del músculo aductor externo a partir del tamaño y profundidad de las fosas temporales y de la extensión y grado de impresión de la aponeurosis interna. Dicha estimación sugiere un grado de desarrollo comparable al de las especies actuales del género.

Las únicas especies fósiles cuyos hallazgos incluyen cráneos suficientemente completos son Marplesornis novaezealandiae del Plioceno de Nueva Zelanda (Marples, 1960) y Paraptenodytes antarcticus del Mioceno temprano de Patagonia (Simpson, 1946). Estas presentan características similares con Spheniscus, como ya se mencionó al inicio de la discusión, pero también presentan caracteres que lo diferencian.

Para el caso de Marplesornis, Marples (1960: 188) menciona que M. novaezealandiae posee un articular robusto y no interrumpido o recortado entre sus procesos interno y postarticular. Zusi (1975: 80) añade que el proceso postarticular es corto y grueso.

Por su parte, P. antarcticus se diferencia de Spheniscus por tener los procesos opistóticos bífidos y el proceso postarticular corto (Simpson, 1946: 11). Para el segundo carácter, $S$. megaramphus presenta su proceso postarticular de una forma intermedia entre $P$. antarcticus (muy corto) y Spheniscus actuales (muy largo).

Lamentablemente, la elongación de los picos de estas especies fósiles es desconocida, pero su movilidad mandibular y desarrollo del músculo pterigoideo en ambas era limitado. El desarrollo de este último no puede apreciarse en el cráneo de $S$. 
megaramphus porque carece de pterigoides, sin embargo su inserción en el proceso postarticular da una idea de que su tamaño pudo ser grande.

Para la definición de especie se descarta la posibilidad de que se trate de $S$. predemersus, ya que este presenta dimensiones mucho menores para los pocos elementos post-craneales descriptos, a las que se esperan para $S$. megaramphus (ver Simpson, 1971). El material reportado por Walsh \& Hume (2001) como cf. Spheniscus sp. para la formación Bahía Inglesa (Chile) no puede ser comparado con S. megaramphus debido a que se trata de elementos post-craneales.

El hallazgo de los restos aquí descritos y del material de Bahía Inglesa, permiten remontar el biocrón de los Spheniscinae al menos hasta el Mioceno tardío. Un dato importante es que en el nivel Montemar, de donde proviene $S$. megaramphus, también se han encontrado restos post-craneales (62 elementos apendiculares aislados) cuyo estudio preliminar indica que todos pertenecerían al género Spheniscus y que son indiferenciables a nivel infra-genérico.

\section{2. Características morfológicas}

La diferencia entre el holotipo y los paratipos es la misma encontrada para los individuos de $S$. humboldti. Inter-específicamente, sólo $S$. mendiculus se ha podido diferenciar de las otras tres especies actuales, por su reducido tamaño (ver Cuadro 1).

Sin embargo, existen diferencias dimensionales entre el holotipo y el paratipo MUSM 363, a nivel de la longitud, alto del pico y longitud de la cabeza (neurocráneo). Para las dos primeras medidas MUSM 363 representa cerca de 78 y $85 \%$ respectivamente del holotipo. Para la tercera, el 94\%.

Sobre esto se podría hacer un paralelo con el caso de $S$. humboldti (Zavalaga \& Paredes, 1997: 106) y S. magellanicus (Scolaro et al., 1983: 221) donde existe un ligero dimorfismo sexual. En estas especies, las dos primeras medidas representan para las hembras entre el 86 y $93 \%$ de la longitud del pico de los machos. Para el caso de la longitud de la cabeza (tomada sólo para $S$. humboldti), la medida en las hembras es el $94 \%$.

En los dos primeros casos las proporciones en los actuales son un poco mayores, sin embargo hay que considerar la diferencia proporcional en el largo de los picos entre las especies actuales y los fósiles; en la tercera, donde no hay diferencia entre fósiles y actuales, la proporción es la misma.

Esto podría sugerir que el holotipo fue un individuo macho, mientras que el paratipo MUSM 363 una hembra de S. megaramphus.

De acuerdo a la interpretación de los índices propuestos por Zusi (1975: 67) calculados en el presente trabajo (ver Cuadro 1), es probable que S. megaramphus estuviera adaptado para la captura de presas de gran tamaño, más que cualquiera de los Spheniscinae actuales. Este nivel de adaptación fue posible al combinar un grado de elongación del pico (índice $\mathrm{N}^{\circ}$ 3) superior al de Aptenodytes patagonica, con una movilidad mandibular (índice $\mathrm{N}^{\circ} 4$ ) que corresponde a los valores medios de S. humboldti y S. magellanicus y mucho mayor al de la especie antártica. Ambos géneros 
son reconocidos por sus capacidades para la captura de grandes presas como peces y calamares (Zusi, 1975: 78). A estos caracteres mencionados se suman los exclusivos del género, tales como el gran desarrollo del músculo aductor externo, la presencia del borde tomial, la fuerte constitución del pico y la punta del pico en forma de gancho (Zusi, 1975: 79-80), que en el caso de $S$. megaramphus es más desarrollada que en los actuales.

El tamaño de la glándula salina se relaciona directamente con la cantidad de sales ingerida por el ave, la que a su vez depende de los hábitos alimenticios y del grado de oceanidad (Schmidt-Nielsen, 1960).

En $S$. megaramphus, la glándula salina alcanzó un mayor desarrollo que en $S$. humboldti y las demás especies del género. Puesto que su morfología sugiere que se alimentaba preferentemente de peces como los representantes vivientes del género, dicho carácter podría relacionarse con el tiempo de permanencia en el agua, pudiendo inferirse que esta especie pasaba mucho tiempo nadando o realizaba recorridos de larga distancia. Este último comportamiento le habría sido de utilidad si mar templado - cálido propuesto para el Mioceno de la costa del Pacifico sudoeste (Cione \& Tonni, 1981; Muizon \& DeVries, 1985) sólo alcanzaba niveles de productividad suficientes durante determinada época del año. La productividad fluctuante durante el año sería provocada por el avance creciente de corrientes marinas frías, que finalmente condujo al descenso de las temperaturas marinas en el Plioceno temprano (Olson, 1983: 401).

\section{Agradecimientos}

Nuestro más profundo agradecimiento a los señores Ana María y Santiago E. Stucchi, Carlos Zavalaga, Rodolfo Salas, Thomas DeVries, Luis Coloma, Judith Figueroa, Claudia Tambussi, RichardZusi, Alejandro Simeone, Juan Carlos Torres, Jorge Noriega, Piotr Jadwiszczak, Martin Chávez, Falco Rivera, Julia Tejada y Dan Omura.

\section{Referencias citadas}

ACOSTA, C., FRITIS, O., TAMBUSSI, C. \& QUINZIO, A., 2002 - Nuevos restos de pingüinos (Aves: Spheniscidae) en la Formación Bahía Inglesa (Mioceno superior - Plioceno inferior) de Chile. $1^{o}$ Congreso Latinoamericano de Paleontología de Vertebrados. Santiago de Chile

CIONE, A. \& TONNI, E., 1981 - Un Pingüino De La Formación Puerto Madryn (Mioceno tardío) De Chubut, Argentina. Comentarios Acerca Del Origen, la Paleoecología y Zoogeografía de los Spheniscidae. Anais II Congresso Latinoamericano Paleontología, Porto Alegre: 591-604.

MAROCCO, R. \& MUIZON, C. de, 1988 - Los Vertebrados del Neógeno de la Costa Sur del Perú. Bulletin de l'Institut Français d'Études Andines, 27(2): 105-117; Lima: Instituto Francés de Estudios Andinos.

MARPLES, B. J., 1960 - A Fossil Penguin From The Late Tertiary Of North Canterbury. Records of the Canterbury Museum, Vol. VII, N ${ }^{\circ}$ III: 185-195. 
MUIZON, C. de \& DEVRIES, T., 1985 - Geology and paleontology of late Cenozoic marine deposits in the Sacaco area (Peru). Geolische Rundschau, 74(3): 547-563; Stuttgart.

MYRCHA, A., JADWISZCZAK, P., TAMBUSSI, C., NORIEGA, J., GAZDZICKI, A., TATUR, A. \& DEL VALLE, R., 2002 - Taxonomic revisión of Eocene Antarctic penguins based on Tarsometatarsal Morphology. Polish Polar Research, Vol. 21, $\mathbf{N}^{\mathbf{0}} \mathbf{1}$ : $5-46$.

NORIEGA, J. \& TAMBUSSI, C. P., 1989 - Un Spheniscidae (Aves, Sphenisciformes) del Mioceno tardío de la Costa Sur del Perú. Resumen de la VII Jornada Argentina de Paleontología de Vertebrados. Ameghiniana, 26(3-4): 247.

OLSON, S., 1983 - Fossil Seabirds and Changing Marine Eviroments in the Late Tertiary of South Africa. South African Journal of Science, Vol. 79: 399-402; Cape Province, South Africa.

SCOLARO, J., HALL, M. \& XIMÉNEZ, I., 1983 - The Magellanic Penguin (Spheniscus magellanicus): Sexing Adults by Discriminant Analysis of Morphometric Characters. The Auk, 100(1): 221 - 224.

SCHMIDT - NIELSEN, K., 1960 - Fisiología animal. Adaptación y medio ambiente; Barcelona - España: Ediciones Omega S.A.

SIMPSON, G. G., 1946 - Fossil Penguins Publications of The Scarrit Expeditions, number 33. Bulletin of the American Museum of Natural History, Vol. 87: 1-100; New York.

SIMPSON, G. G., 1971 - Fossil Penguin from the Late Cenozoic of South Africa. Science, Vol. 171: 1144 - 1145.

SIMPSON, G. G., 1976 - Penguins Past and Present, Here and There, 150p.; New Haven London: Yale University Press.

TAMBUSSI, C., REGUERO, M., MARENSSI, S. \& SANTILLANA, S., 2002 - The earliest know penguin and the evolution of Spheniscid size. $1^{\circ}$ Congreso Latinoamericano de Paleontología de Vertebrados. Santiago de Chile.

TONNI, E . \& TAMBUSSI, C.P., 1986 - Las Aves del Cenozoico de la República Argentina. In: IV Congreso Argentino de Paleontología y Bioestratigrafía, 2: 131-142.

WALSH, S. \& HUME, J., 2001 - A new neogene marine avian assemblage from north-central Chile. Journal of Vertebrate Paleontology, 21(3): 484 - 491.

ZAVALAGA, C. \& PAREDES, R., 1997 - Sex determination of adult Humbodlt Penguins using morphometric characters. Journal of Field Ornithology, 68(1): 102 - 112.

ZUSI, R., 1975 - An interpretation of skull structure in penguins. In: The Biology of Penguins. (B. Stonehouse ed.): 59-84; Macmillan, London. 
Institut Français d'Études Andines - Institut de Recherche pour le Développement (IRD) - PLURAL Editores Pedidos: IFEA, Casilla 18-1217, Lima 18 - Perí, Tel. 4476070 Fax: 4457650 - E-mail: postmasterlifea.org.pe

Web: http://www.ifeanet.org 